\title{
Performance Study of Star Topology in Small Internetworks
}

\author{
Nurul Absar \\ Assistant Professor \\ Department of Computer \\ Science and Engineering, BGC \\ Trust University Bangladesh, \\ Chandanaish, Chittagong, \\ Bangladesh.
}

\author{
Mohammad Jahangir \\ Alam \\ Assistant Professor \\ Department of Computer \\ Science and Engineering \\ Southern University \\ Bangladesh
}

\author{
Tasnuva Ahmed \\ Lecturer \\ Department of Computer \\ Science and Engineering \\ Southern University \\ Bangladesh
}

\begin{abstract}
This paper studies the performance of star topology in a Small Internetworks. In this network model, general LANs models are used and a simulated environment is formed where many applications are in used at a time and their mutual effects thereof. I was performed simulations using OPNET IT GURU Academic Edition simulator. Several simulation graphs were obtained and used to analyze the network performance. The results being obtained represent the optimum possible improvements in terms of number of node, Ethernet delay (second), load (bits/sec). The result was found that when the number of nods and simulation time were varied the server loads also was changed but the delay was almost same. The only limitation of this program is that we can not save translated text i.e it can only work with predetermined application. It can apply as a University network which covers most of its departments and colleges, many industries and garments, etc. A detailed simulation study helped to find out the best solution of research questions.
\end{abstract}

\section{Keywords}

Small Internetworks, OPNET IT GURU, Ethernet delay, Ethernet node, Ethernet load. Star topology.

\section{INTRODUCTION}

A computer network is simply two or more computers connected together so they can exchange information [1]. A small network can be as simple as two computers linked together by a single cable. Internetworking-the communication between two or more networks-encompasses every aspect of connecting computers together [2]. A physical topology is the physical layout, or pattern, of the nodes on a network. In the computer networking world the most commonly used topology in LAN is the star topology. Star topologies can be implemented in home, offices or even in a building. All the computers in the star topologies are connected to central devices like hub, switch or router. The failure of each node or cable in a star network, won't take down the entire network as compared to the Bus topology. The main purpose of a campus network is efficient resource sharing and access to information among its users [3].A star topology is designed with each node (file server, workstations, and peripherals) connected directly to a central network hub or concentrator (Fig.1).Data on a star network passes through the hub or concentrator before continuing to its destination. The hub or concentrator manages and controls all functions of the network. It also acts as a repeater for the data flow. This configuration is common with twisted-pair cable; however, it can also be used with coaxial cable or fiber-optic cable

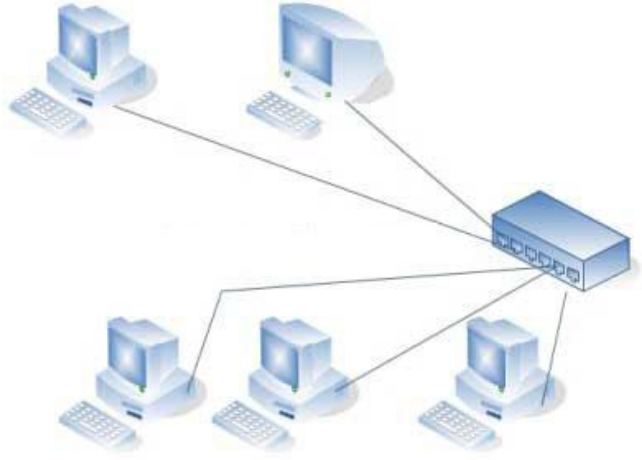

Fig. 1: Star topology [4]

A star configuration is simple: Each of several devices has its own cable that connects to a central hub, or sometimes a switch, multipoint repeater, or even a Multistation Access Unit (MAU). Data passes through the hub to reach other devices on the network. Ethernet over unshielded twisted pair (UTP), whether it is 10BaseT, 100BaseT, or Gigabit, all use a star topology.

Star networks are one of the most common computer network topologies. In its simplest form, a star network consists of one central switch, hub or computer which acts as a router to transmit messages. If the central node is passive, the originating node must be able to tolerate the reception of an echo of its own transmission, delayed by the two-way transmission time (i.e. to and from the central node) plus any delay generated in the central node. An active star network has an active central node that usually has the means to prevent echo-related problems.

The star topology reduces the chance of network failure by connecting all of the systems to a central node. When applied to a bus-based network, this central hub rebroadcasts all transmissions received from any peripheral node to all peripheral nodes on the network, sometimes including the originating node. All peripheral nodes may thus communicate with all others by transmitting to, and receiving from, the central node only. The failure of a transmission line linking any peripheral node to the central node will result in the isolation of that peripheral node from all others, but the rest of the systems will be unaffected.

\section{AIMS AND OBJECTIVE}

The main goal of this research work is to briefly the design and implementation of Small Inter Network for star topology. 


\section{SCOPE OF THE PAPER}

This research work reports on how to design a small internetworks, Creating different scenarios and the performance analysis of star topology are achieved. In what follows to give the descriptive idea about the small internetworks mechanism and their functionality but doesn't provide any deep information regarding the architecture of those mechanisms but how data is actually transferred in a network as opposed to its physical design. Topology can be considered as a virtual shape or structure of a network. In this research, analysis of internetworks to measure the performance over star topology have been done in a simple and understandable fashion so that it might be helpful for those who have some intention to do further research.

\section{RESEARCH METHODOLOGY}

We divide our work into two parts. First of all, we design small internetworks model with star topology, simulate it by using OPNET and observe the performance for different scenarios based on Load delay, delay variation, on different links as well as nodes. Secondly, in the same network scenario, we implement second floor. We employ Optimized Network Engineering Tool (OPNET) as a simulator to evaluate and to analyze the comparative performance of this topology. The methodology adopted in this modeling and simulation experiment is presented in the OPNET architecture algorithmic diagram that is shown in Fig.2 with slight modifications as described below [5]:

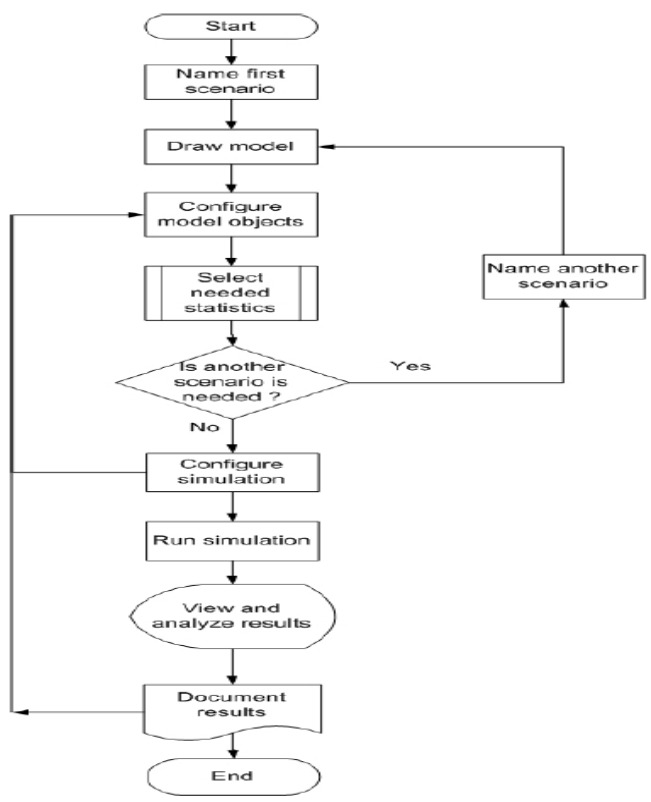

Fig. 2: The OPNET Architecture

\section{OPNET OVERVIEW}

OPNET's IT Guru provides a Virtual Network Environment that models the behavior of an entire network, including its routers, switches, protocols, servers, and individual applications. By working in the Virtual Network Environment, IT managers, network and system planners, and operations staffs are empowered to diagnose difficult problems more effectively, validate changes before they are implemented, and plan for future scenarios including growth and failure [6]. OPNET is a discrete network simulator which contains a comprehensive development environment supporting the modeling and performance evaluation of communication networks and distributed systems [7].
Designing an efficient network plays an important role in this world and then it is even essential part to check the performance of the designed network, which will be a difficult task in a real time application. For this many network simulators have been designed so far among the most reputed are OPNET (Optimized Network Engineering Tool) Modeler and NS2 (Network Simulator). OPNET modeler is not an open source product it needs license to access it provides GUI and consists of predefined models, protocols and algorithms and supports with lot of documentation it is specially used for commercial purpose. NS2 is an open source simulating tool it is combination of $\mathrm{C}++\&$ Otcl with less document support specially used by developers.

We are using the Optimized Network Engineering Tool (OPNET v 9.1) software for our simulations. OPNET is a network simulator. It provides multiple solutions for managing networks and applications e.g. network operation, planning, research and development (R\&D), network engineering and performance management. OPNET 9.1 is designed for modeling communication devices, technologies, protocols and to simulate the performance of these technologies. OPNET plays a key role in today's emerging technical world in developing and improving the wireless technology protocols such as WiMAX, WiFi, UMTS, etc, design of MANET routing protocols, working on new power management systems over sensor networks and enhancement of network technologies such as Ipv6, MPLS etc. Working of OPNET generally divided into four parts, model design, applying statistics, run simulation and then to view results and to analyze the results, if the results are not correct then it has to be re-modeled and then to apply new statistics.

\section{CONCEPT OF COMPONENTS}

This section discusses about the following network components used in the suggested network models running on OPNET [8]. All network components have been described according to the instruction OPNET IT GURU Academics edition 9.1 versions [8]. An internetwork is designed by using some basic components:

- Hubs (concentrators)

- Bridges

- Switches

- Routers

A representation of a real-world network objects that can transmit and receive information. Node domain is an internal infrastructure of the network domain. Node can be routers, workstations, satellite and so on. A communication medium that connects nodes to one another. Links can represent electrical or fiber optic cables.

\subsection{Ethernet}

Ethernet is a family of computer networking technologies for local area networks (LANs). Ethernet was commercially introduced in 1980 and standardized in 1985 as IEEE 802.3. Ethernet has largely replaced competing wired LAN technologies. The Ethernet standards comprise several wiring and signaling variants of the OSI physical layer in use with Ethernet. The original 10BASE5 Ethernet used coaxial cable as a shared medium. Later the coaxial cables were replaced by twisted pair and fiber optic links in conjunction with hubs or switches. Data rates were periodically increased from the original 10 megabits per second to 100 gigabits per second. 
Systems communicating over Ethernet divide a stream of data into shorter pieces called frames. Each frame contains source and destination addresses and error-checking data so that damaged data can be detected and re-transmitted. As per the OSI model Ethernet provides services up to and including the data link layer.

\subsection{Sm_Int_wkstn}

The ethernet_wkstn_adv node model represents a workstation with client-server applications running over TCP/IP and UDP/IP. The workstation supports one underlying Ethernet connection at $10 \mathrm{Mbps}, 100 \mathrm{Mbps}$, or $1000 \mathrm{Mbps}$.

This workstation requires a fixed amount of time to route each packet, as determined by the "IP Forwarding Rate" attribute of the node. Packets are routed on a first-come-first-serve basis and may encounter queuing at the lower protocol layers, depending on the transmission rates of the corresponding output interfaces.

Protocols: RIP, UDP, IP, TCP, IEEE 802.3 (Ethernet, Fast Ethernet, Gigabit

Ethernet), OSPF

Port Interface Description: 1 Ethernet connection at 10 Mbps, 100 Mbps or 1000 Mbps

\subsection{C_SSII_1100_3300_4s_ae52_e48_ge3}

This node is a stack of two 3Com SuperStack II 1100 and two Superstack II 3300 chassis (3C_SSII_1100_3300) with four slots (4s), 52 auto-sensing Ethernet ports (ae52), 48 Ethernet ports (e48), and 3 Gigabit Ethernet ports (ge3). Three of the above four chassis are equipped with an optional one-port 1000BaseX Gigabit Ethernet module. The model represents only the Layer-2 functionalities of the devices. The stack configuration is as follows.

\subsection{Application Config}

The "Application Config" node can be used for the following specifications:

1. "ACE Tier Information": Specifies the different tier names used in the network model. This attribute will be automatically populated when the model is created using the "Network->Import Topology->Create from ACE." option. The tier name and the corresponding ports at which the tier listens to incoming traffic are cross-referenced by different nodes in the network.

2. "Application Specification": Specifies applications using available application types. You can specify a name and the corresponding description in the process of creating new applications. For example, "Web Browsing (Heavy HTTP 1.1)" indicates a web application performing heavy browsing using HTTP 1.1. The specified application name will be used while creating user profiles on the "Profile Config" object.

3. "Voice Encoder Schemes": Specifies encoder Parameters for each of the encoder schemes used for generating Voice traffic in the network.

\subsection{Sm_Int_server}

The ethernet_server_adv model represents a server node with server applications running over TCP/IP and UDP/IP. This node supports one underlying Ethernet connection at 10 Mbps, 100 Mbps, or 1 Gbps. The operational speed is determined by the connected link's data rate. The Ethernet MAC in this node can be made to operate either in full-duplex or half-duplex mode. Note that when connected to a Hub, it should always be set to "Half Duplex". A fixed amount of time is required to route each packet, as determined by the "IP Forwarding Rate" attribute of the node. Packets are routed on a FCFS basis and may encounter queuing at the lower protocol layers, depending on the transmission rates of the corresponding output interface.

Protocols: RIP, UDP, IP, TCP, Ethernet, Fast Ethernet, Gigabit Ethernet, OSPF

Interconnections: 1 Ethernet connection at $10 \mathrm{Mbps}, 100$ Mbps, or $1000 \mathrm{Mbps}$

Attributes: Ethernet Operational Mode: Specifies the mode in which the Ethernet MAC operates (Half Duplex or Full Duplex)

\subsection{Sm_Profile_Config}

The "Profile Config" node can be used to create user profiles. These user profiles can then be specified on different nodes in the network to generate application layer traffic. The application defined in the "Application Config" objects is used by this object to configure profiles. Therefore, you must create applications using the "Application Config" object before using this object. We can specify the traffic patterns followed by the applications as well as the configured profiles on this object.

\subsection{CS_2514_1s_e2_sl2}

The CS_2514_1s_e2_sl2 model represents the following device:

\section{Vendor: Cisco Systems \\ Product: $\quad$ CISCO2514}

Device Class: Router

Configuration: Ethernet to Ethernet using IP via serial IP WAN

General Operation: This model represents an IP-based router/gateway model supporting two $10 \mathrm{Mbps}$ ethernet hub interfaces and two serial IP interfaces at selectable data rates. IP packets arriving on an IP interface are routed to the appropriate output interface based on their destination IP address. The Routing Information Protocol (RIP) or Open Shortest Path First (OSPF) protocol may be used to automatically and dynamically create the routing tables and select routes in an adaptive manner. The key model features are:

1. An IP forwarding rate of 2000 packets/sec - this estimated value is based on Cisco System's online documentation/product guide (02/97)

2. The router model implements a "store and forward" type of switching methodology.

A typical use of this device is to route data between two ethernet LAN segments connection via an IP network.

\section{Interconnections:}

1. Two $10 \mathrm{Mbps}$ ethernet hub connections.

2. Two serial IP interfaces at selectable data rates.

\subsection{BaseT}

The 10BaseT duplex link represents an Ethernet connection operating at $10 \mathrm{Mbps}$. It can connect any combination of the nodes (except Hub-to-Hub, which cannot be connected) which are 1) Station 2) Hub 3) Bridge 4) Switch 5) LAN nodes and whose Packet Formats and Data Ra are: Ethernet and $10 \mathrm{Mbps}$ respectively. 


\section{RESULT AND DISCUSSIONS}

A comparative analysis of a small internwtwork for star topology with different number of nodes, expand the network, taking object statistics, server Load (bits/sec), global statistics Delay (sec) or from the entire network have been presented in this paper. Basically two types of statistics are used for our designed model. Those are1) Global or scenario-wide statistics 2) Object statistics. Global statistics collected from the whole network model designed and the object statistics collected over nodes. These statistics can be applied to a network model based on the user requirement for this design.

There are three network models, which are configured and run for the same statistics. The basic network is designed with 10 , 20 and 30 work stations connected to a server. Then the extension is made to the network. The global Parameter selected is Delay (sec) and the local parameter selected is Load (bits/sec)

\subsection{Simulated Results}

The following figure illustrated the small internetwork for 10 , 20 and 30 workstations which were designed to measure the performance for star topology as shown in Fig. 7.1, Fig. 7.7, and Fig. 7.11 respectively. Fig. 7.2 (a) to Fig. 7.5 (b) illustrates the comparable performance of the star topology under the different network scenarios measured for the Ethernet Delay (Sec) Vs Ethernet Load (bits/sec) for10 workstations. The number of nodes in second expanded network is varied and the results are compared for different number of nodes. Various expansions are made to the network and then the results are compared. For the first run the expansion is taken with 10 nodes and the Load and delay graph is obtained as in Figure 7.2(a) - 7.5(b). Comparison graph can also be obtained for the two networks, basic and the expanded network consisting of 10 nodes as shown in Figure 7.3(a) an.Similarly the number of nodes can be varied and a comparison can be drawn in the form of graphs. We have taken the total of 20 and 30 workstations connected through a switch. This switch is connected to the server. Server sent the information to the users in bits/sec. All the stations \& server were connected through the $10 \mathrm{BaseT}$ cables. The network designed and simulated result for 20 and 30 workstations connected through a switch have been shown in Figure 7.8(a) -7.10 (b) and Figure 7.12(a) - 7.15(b) respectively. d (b).

All analysis has been done to the procedure of $[9, \mathbf{1 0}]$. On the basis of these simulated results a table is prepared to study the comparison of load on server (Bits /Second) and average delay (Sec) depends on amongst all the scenarios which is shown in Table 1.0.

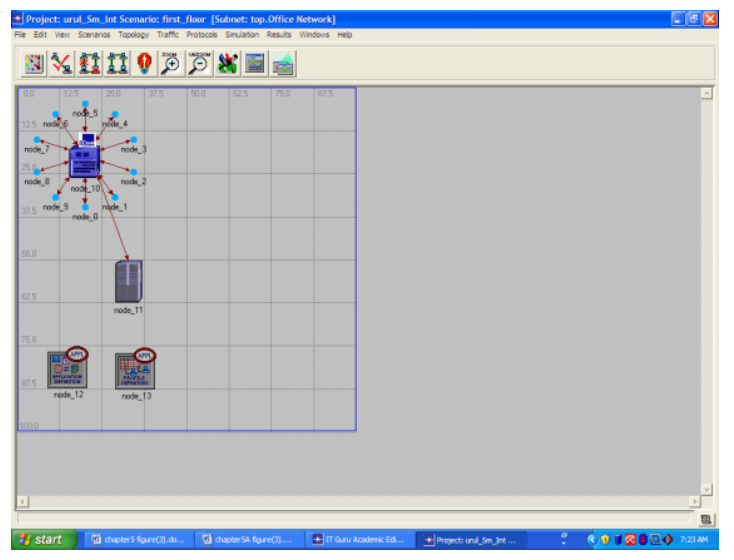

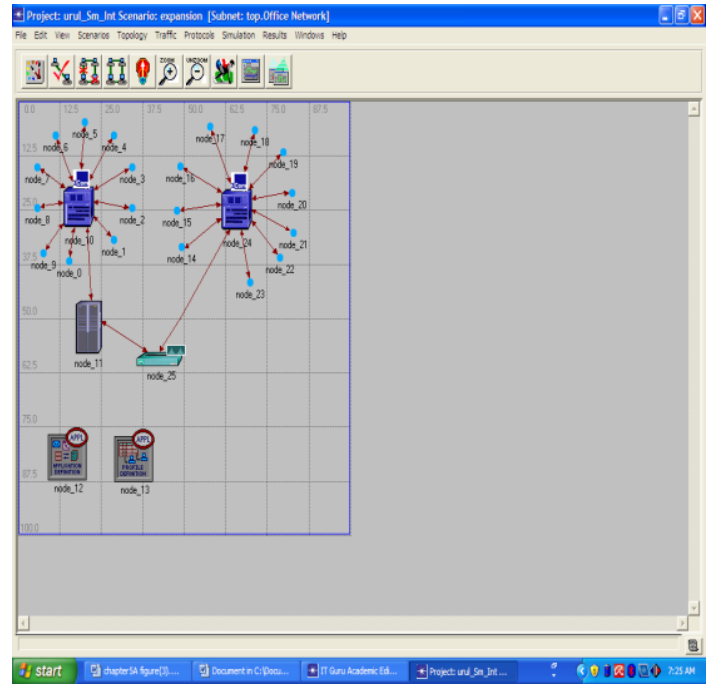

Figure 7.1: Scenario with expanded network for 10 Work Station.

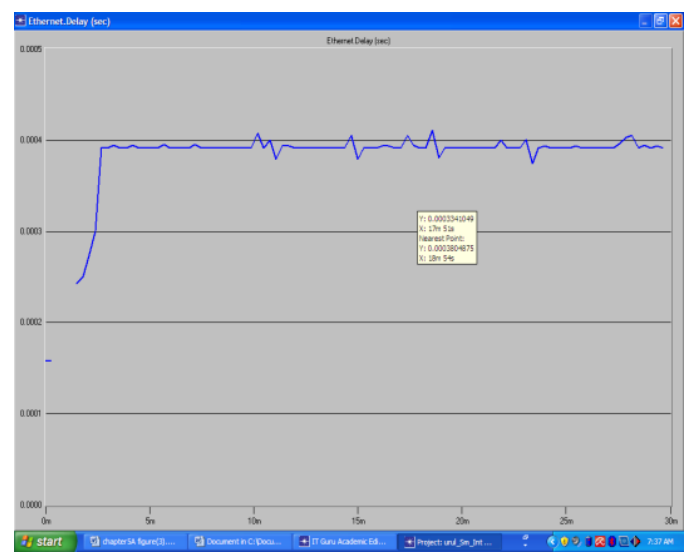

(a)

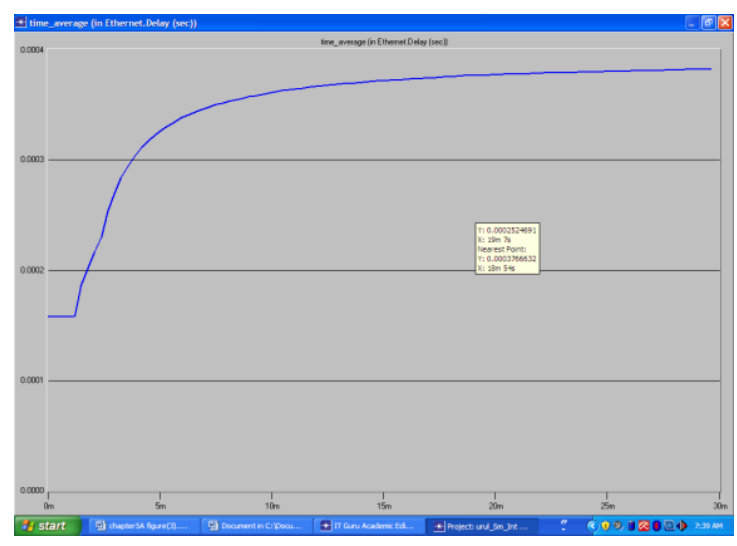

(b)

Figure 7.2: (a) Ethernet Delay (Sec) (b) and Time_ Average (in Ethernet Delay (Sec)) first floor for 10 nodes (Global Statistics). 


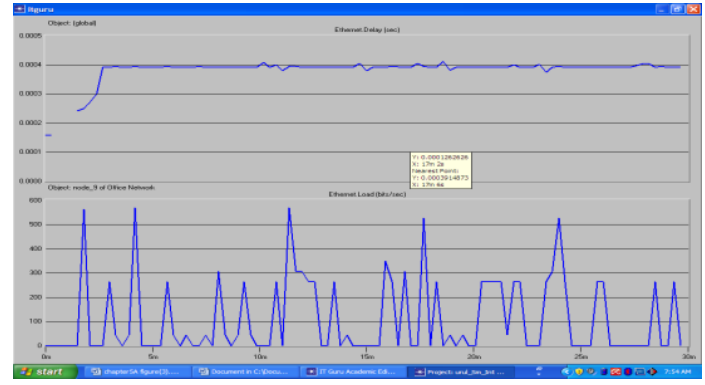

(a)

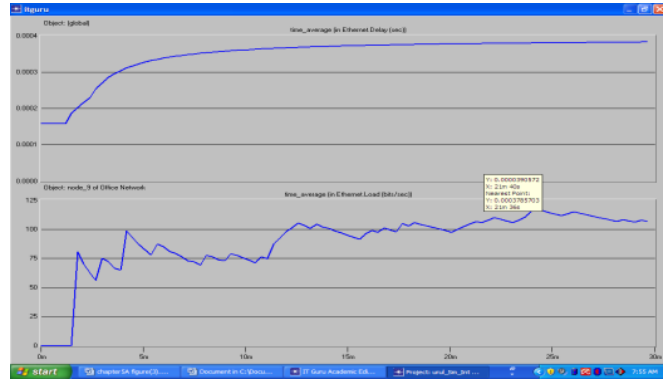

(b)

Figure 7.3: Ethernet Delay (Sec) Vs Ethernet Load (bits/sec)first floor for Object nodes_9 of office network and (b)time _average (in Ethernet Delay (Sec)) Vs Ethernet time_average (in Load (bits/sec)) first floor for Object nodes_9 of office network (Global Statistics Vs Object statistics)

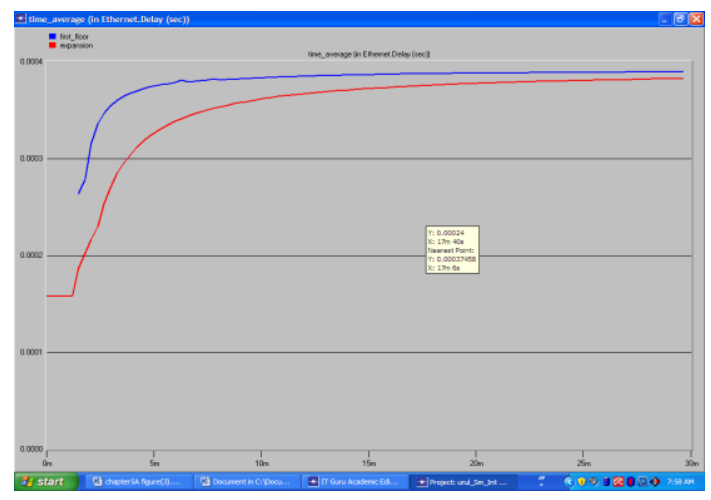

(a)

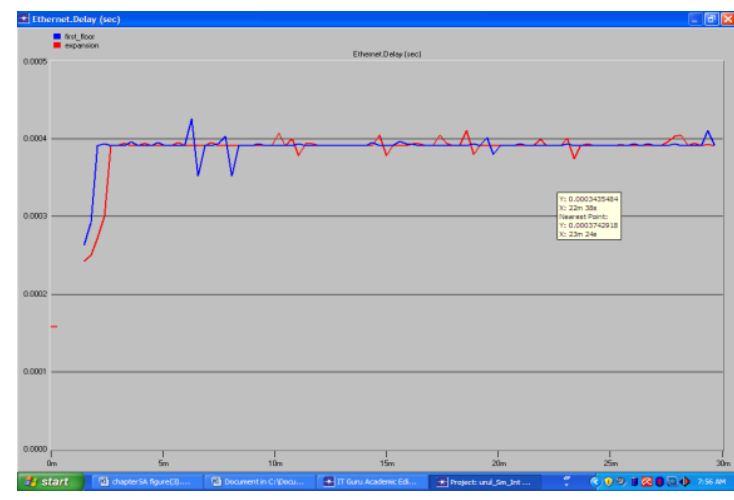

(b)

Figure 7.4: (a) Comparison of Ethernet Delay (Sec) between Scanerio1 and expansion (first floor and second floor for 10 nodes) and (b) Comparison of time_average (in Ethernet Delay (Sec)) between Scanerio1 and expansion (first floor and second floor for 10 nodes (Global Statistics)).

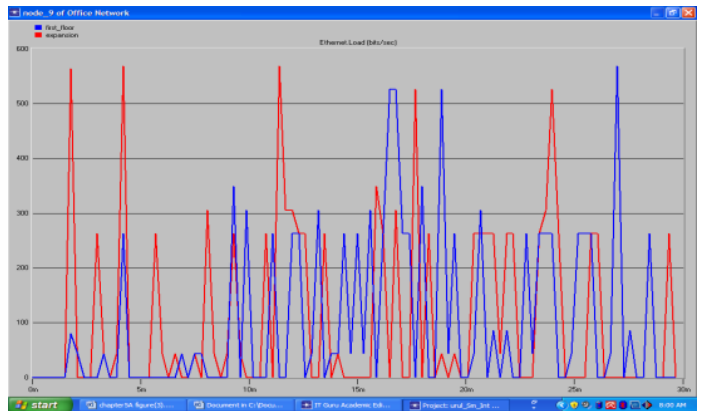

(a)

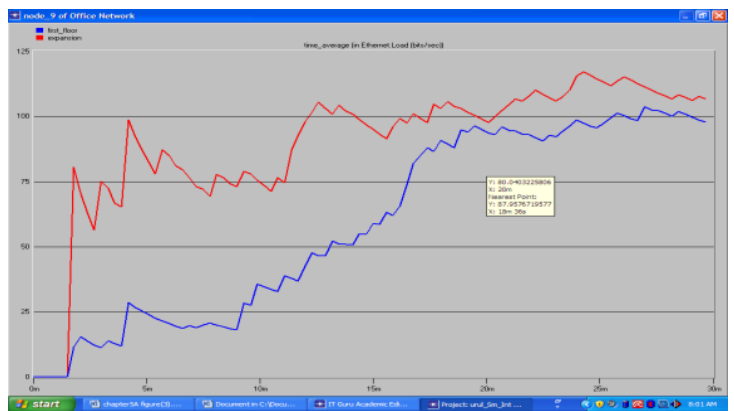

(b)

Figure 7.5: (a) Comparison of Ethernet Load (bits/Sec) between Scanerio1 and expansion (first floor and second floor for 9th node) and (b) Comparison of time average_( in Ethernet Load ( bits/Sec)) between Scanerio1 and expansion (first floor and second floor for 9th node (Object Statistics)).

\subsubsection{Simulated Results for 20 work stations}

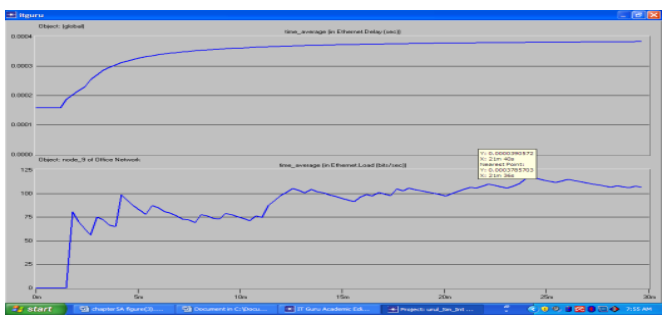

(a)

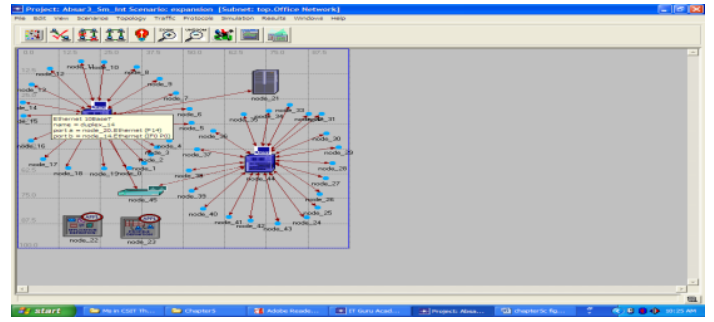

(b)

Figure 7.7: (a) Ethernet Delay (Sec) first floor for 20 Work Station.and (b) Time_Average (in Ethernet Delay (Sec)) first floor for 20 Work Station. (Global Statistics). 


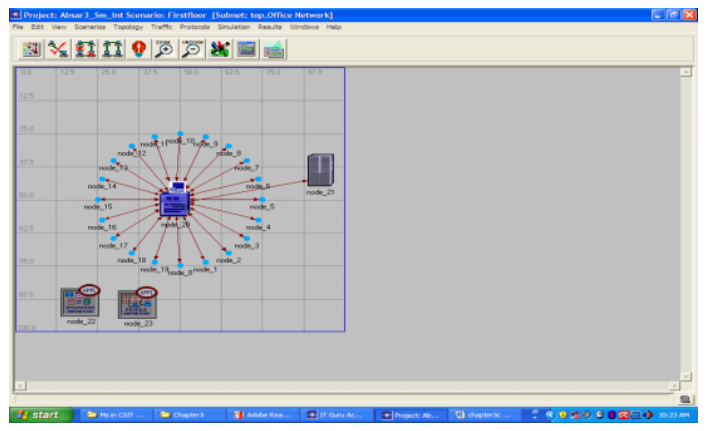

(a)

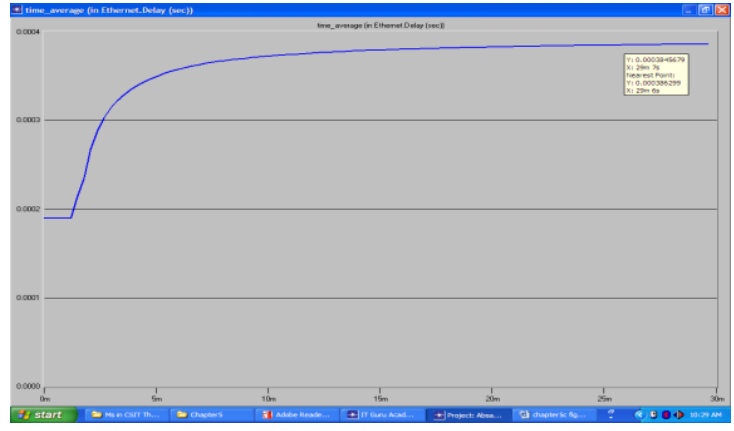

(b)

Figure 7.7: (a) Ethernet Delay (Sec) first floor for 20 Work Station.and (b) Time_Average (in Ethernet Delay (Sec)) first floor for 20 Work Station. (Global Statistics).

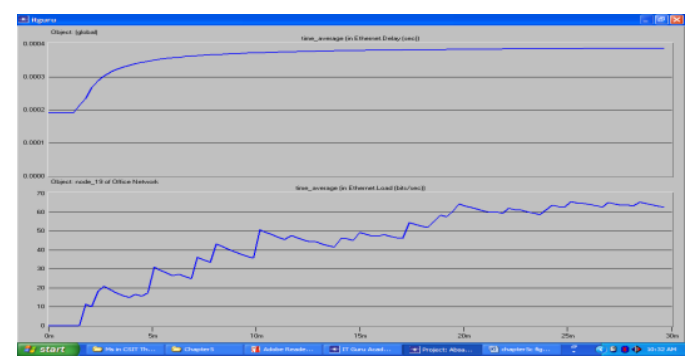

(a)

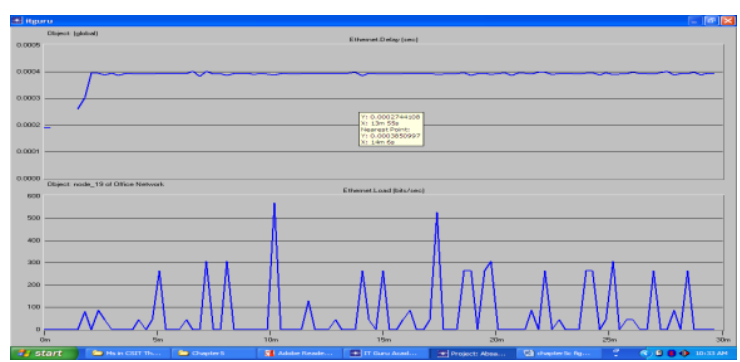

(b)

Figure 7.8: (a) Ethernet Delay (Sec) Vs Ethernet Load (bits/sec)first floor for Object nodes_19 of office network and (b) time _average (in Ethernet Delay (Sec)) Vs Ethernet time_average (in Load (bits/sec)) first floor for Object nodes_19 of office network (Global Statistics Vs Object statistics).

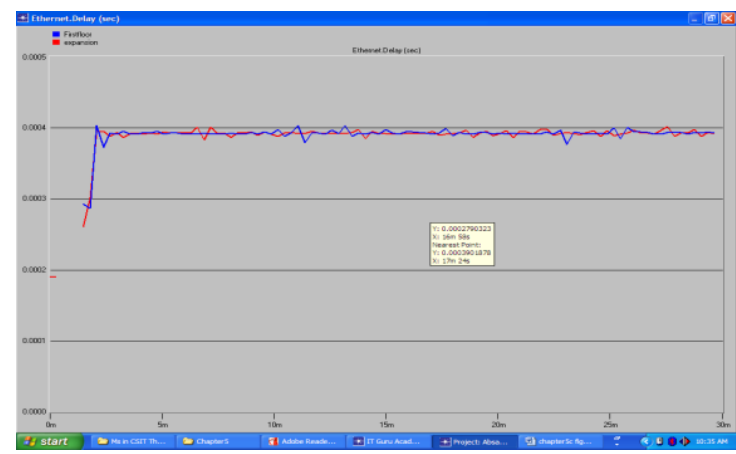

(a)

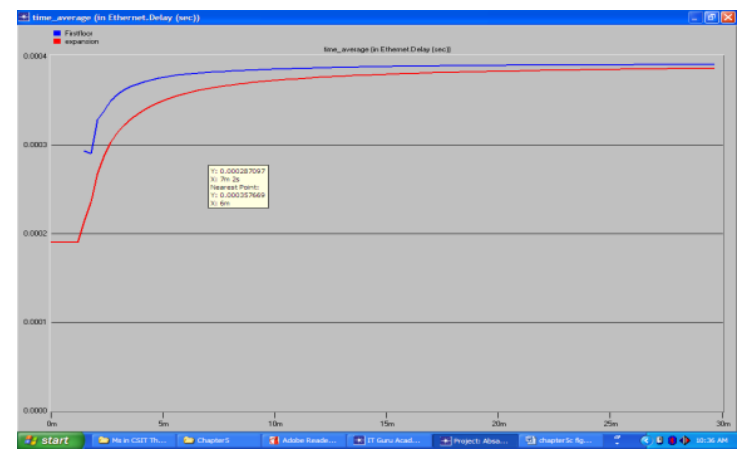

(b)

Figure 7.9: (a) Comparison of Ethernet Delay (Sec) between Scanerio1 and expansion (first floor and second floor for 20 nodes) and (b) Comparison of time_average (in Ethernet Delay (Sec) ) between Scanerio1 and expansion (first floor and second floor for 20 nodes(Global Statistics).

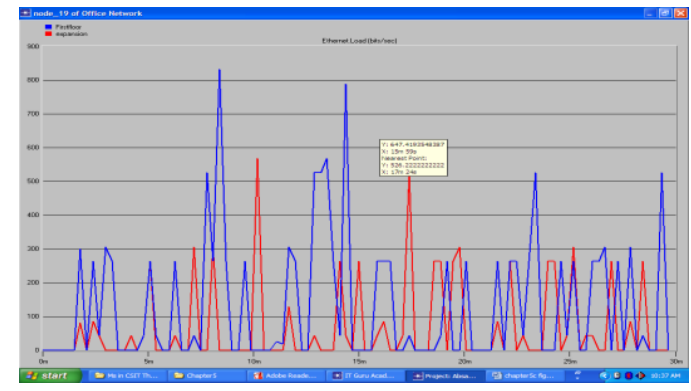

(a)

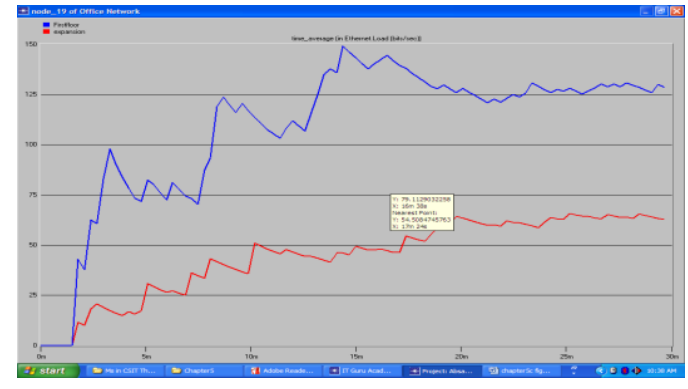

(b)

Figure 7.10: (a) Comparison of Ethernet Load (bits/Sec) between Scanerio1 and expansion (first floor and second floor for 19th node) and (b) Comparison of time average_(in Ethernet Load (bits/Sec)) between Scanerio1 and expansion (first floor and second floor for 19th node (Object Statistics)). 


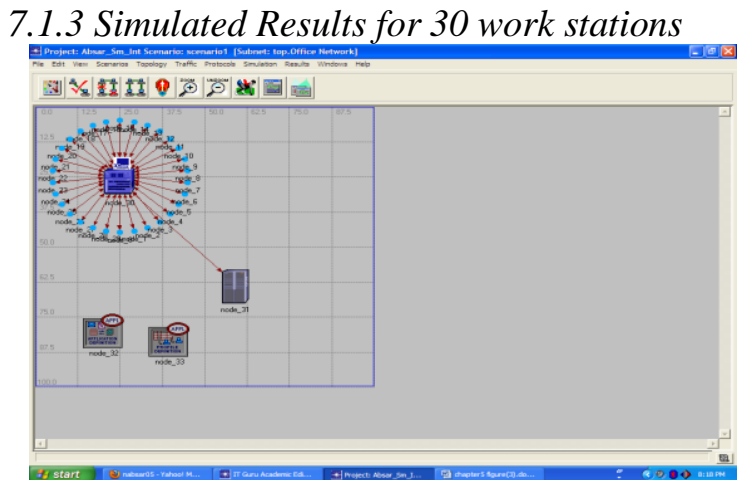

(a)

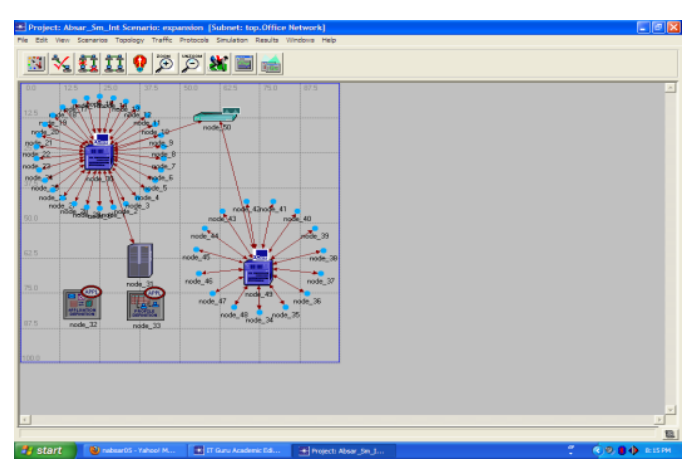

(b)

Figure 7.11: (a) Scenario for first floor for 30 nodes and (b) Scenario with second floor for 20 nodes.

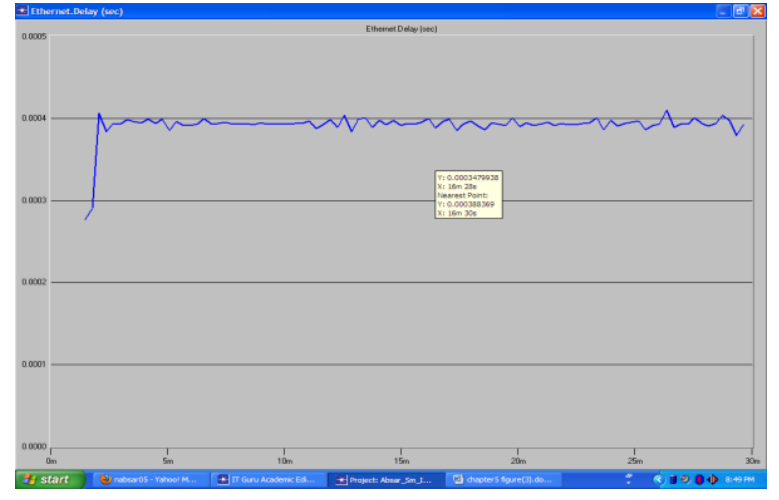

(a)

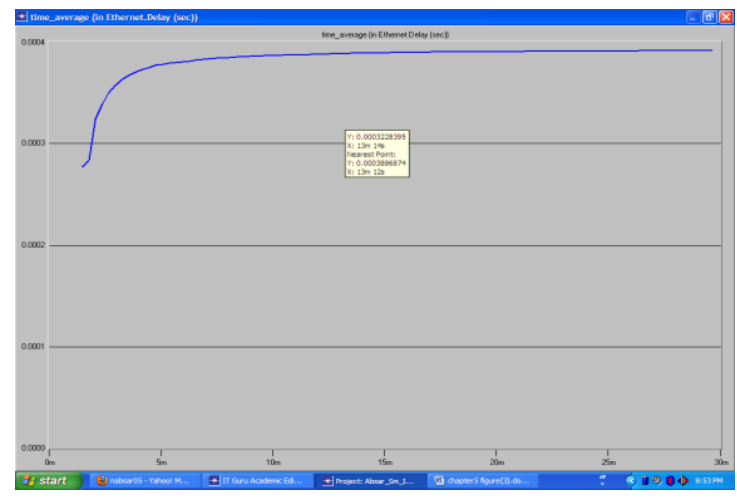

(b)

Figure 7.12: (a) Ethernet Delay (Sec) first floor for 30 nodes and (b) Time_Average (in Ethernet Delay (Sec)) first floor for 30 nodes (Global Statistics).

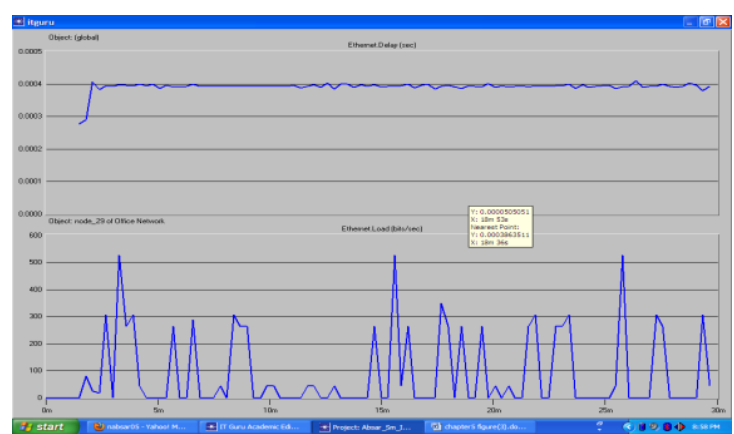

(a)

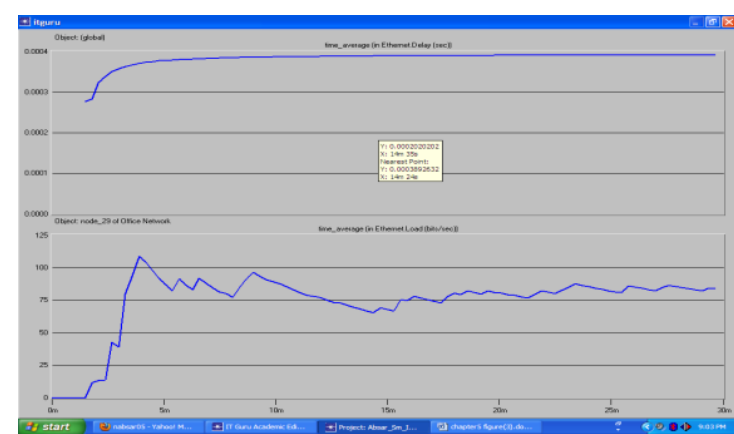

(b)

Figure 7.13: (a) Ethernet Delay (Sec) Vs Ethernet Load (bits/sec)first floor for Object nodes_29 of office network and (b) time _average (in Ethernet Delay (Sec)) Vs Ethernet time_average (in Load (bits/sec)) first floor for Object nodes_29 of office network (Global Statistics Vs Object statistics).

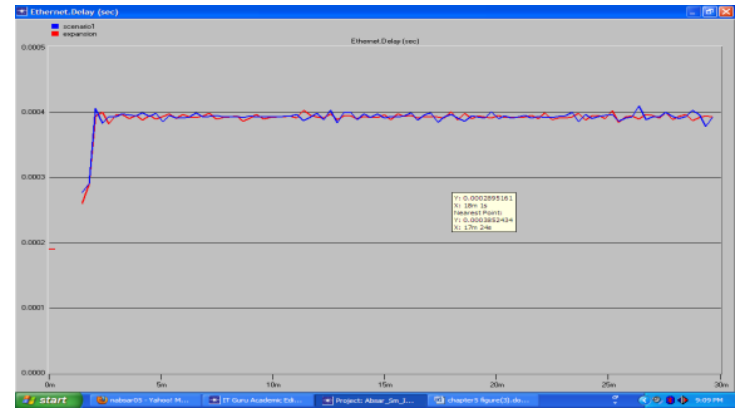

(a)

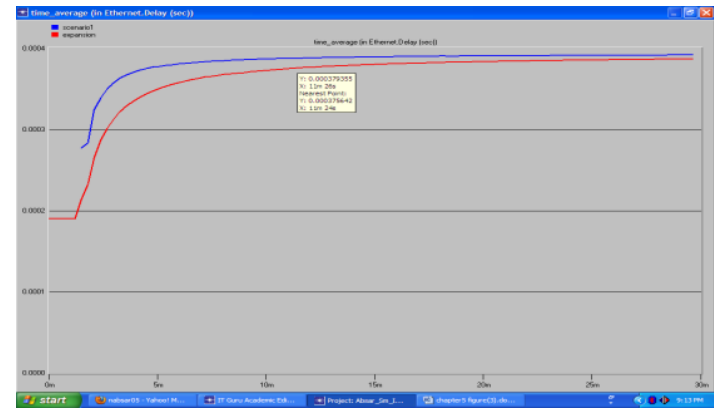

(b)

Figure 7.14: (a) Comparison of Ethernet Delay (Sec) between Scanerio1 and expansion (first floor for 30 nodes and second floor for 20 nodes) and (b) Comparison of time_average (in Ethernet Delay (Sec)) between Scanerio1 and expansion (first floor and second floor for 30 nodes (Global Statistics)). 


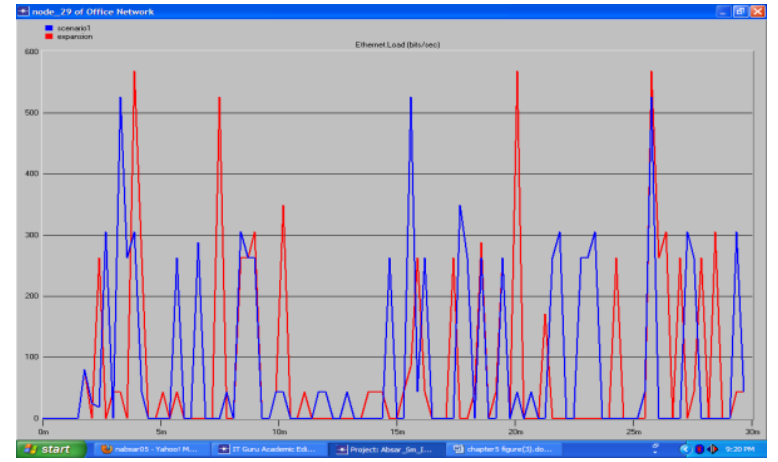

(a)

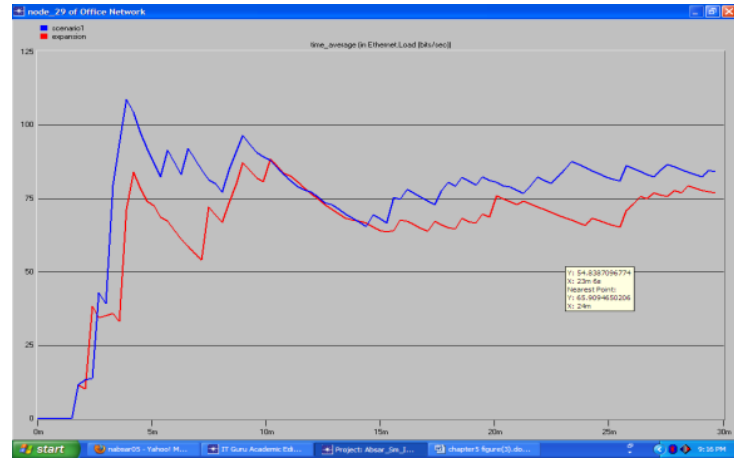

(b)

Figure 7.15: (a) Comparison of Ethernet Load (bits/Sec) between Scanerio1 and expansion (first floor and second floor for 29th node) and (b) Comparison of time average_ (in Ethernet Load (bits/Sec)) between Scanerio1 and expansion (first floor and second floor for 29th node (Object Statistics)).

Table 1: Comparison of Load on Server (Bits /Second) and Average Delay (Sec) app depends on amongst all the scenarios.

\begin{tabular}{|c|c|c|c|}
\hline $\begin{array}{c}\text { Extension in Load( Work } \\
\text { stations) }\end{array}$ & $\begin{array}{c}\text { Simulation } \\
\text { Duration(hours) }\end{array}$ & $\begin{array}{c}\text { Load On } \\
\text { Server(Bits } \\
\text { /Second) }\end{array}$ & $\begin{array}{c}\text { Average } \\
\text { Delay(Sec)app. }\end{array}$ \\
\hline \multirow{3}{*}{10} & 0.5 & $0.00-2312.89$ & 0.0004 \\
\cline { 2 - 4 } & 1.00 & $0.00-1596.44$ & 0.0004 \\
\cline { 2 - 4 } & 5.00 & $0.00-1147.02$ & 0.0004 \\
\hline \multirow{3}{*}{20} & 10.00 & $0.00-1083.02$ & 0.0004 \\
\cline { 2 - 4 } & 0.5 & $0.00-8513.78$ & 0.0004 \\
\cline { 2 - 4 } & 1.00 & $0.00-6246.22$ & 0.0004 \\
\cline { 2 - 4 } & 5.00 & $0.00-4049.78$ & 0.0004 \\
\hline \multirow{3}{*}{30} & 10.00 & $0.00-3828.98$ & 0.0004 \\
\cline { 2 - 4 } & 0.5 & $0.00-9488$ & 0.0004 \\
\cline { 2 - 4 } & 1.00 & $0.00-7138.67$ & 0.0004 \\
\hline & 5.00 & $0.00-4516.27$ & 0.0004 \\
\hline
\end{tabular}

\section{DISCUSSIONS}

The performance of small Internetwork for 10 work station have been shown in Fig. 7.2 to Fig. 7.5 whereas the Fig. 7.7 to Fig. 7.10 and Fig.7.12 to Fig. 7.16 have been shown the simulated result for 20 and 30 work station respectively.

Fig. 7.2, Fig. 7.7 and Fig.7.12 have been represented the base line of Ethernet delay (sec) of first floor for 10, 20 and 30 work station respectively. It was shown that there was a negligible fluctuation occurred for entire simulation time and there were small change due to the change of simulation time and number of nods which was almost same which equal to 0.4 milliseconds.

A comparison study of Ethernet delay (sec) verses Ethernet load(bit/sec) have been done which have been shown in Fig. 7.3, Fig. 7.8 and Fig.7.13 for 10, 20 and 30 work station respectively of first floor. Those graph have been shown, the delay $(\mathrm{sec})$ how to varies due to the change of load (bit/sec) and it was kept standard for further expansions.

Comparisons of Ethernet Delay (Sec) between first floor and expansion networks for different nods have been shown in
Fig. 7.4, Fig. 7.9 and Fig.7.14. It was shown that when the number of nodes increased or decreased in any network, the delay (sec) was increased moderately in the early stage and after that it was almost same. It was also proved that the delay increased or decreased with the number of nodes increased or decreased respectively for entire network.

A Comparison studies have been made of Ethernet Load (bits/Sec) between first floor and expansion networks for a server which is shown in Fig. 7.5, Fig. 7.10 and Fig.7.15. These graphs were taken to determine the variation of load (bits/Sec) between first floor and expansion networks for a server. From these graph we have cleared that server load was varied with the number of nods.

After simulating basic scenario, we were mad a duplicate network from the original network by using a switch with 10 and 20 workstations connected to it. This switch is connected to the switch in original network. By using this network we are extending the load on the server by10 and 20 workstations. Now simulate the project \& then compare the results for original \& duplicate scenarios for load \& delay. Results were compared for different time durations taken as 
0.5 hour, 1 hour, 5 hours, and 10 hours. The comparison table is as shown in Table 1.0. Various values are obtained for different Scenarios As the numbers of nodes are increased in a network the load on the server increases.

\section{LIMITATION}

The Academic Edition has a limitation on the number of events it can simulate. There is a limit to how many hosts can be attached to a single network. The only limitation of this program is that I could not save translated text i.e it can only work with predetermined application

\section{CONCLUSION}

This paper discusses the simulation-based approach to performance study of star topology in a small internetwork. It gives an introduction to the OPNET Modeler simulator and provided supports. And also describes a set of scenarios and metrics that are used to analyze the performance study of star topology in malicious as well as baseline environments. It is found that when the simulation time and number of nodes is increased the server load also increased i,e server load in a small internetwork is not independent of simulation time and number of nodes. With the help of OPNET software we can find out the maximum number of nodes that can be withstand by a server. So before implemented practically we can find out the breakdown value of server

\section{REFERENCES}

[1] Computer Networks. School of Science and Technology, National Open University of Nigeria.
[2] Bobbinpreet, k., (May, 2012) OPNET based Analysis of Star Topology in Small Internetworks International Journal of Electronics \& Data Communication. Volume 1 No. 1.

[3] Atayero, A.A., Alatishe, A.S. and. Iruemi ,J.O., Modeling and Simulation of a University LAN in OPNET Modeller Environment Department of Electrical and Information Engineering, Covenant University, Nigeria

[4] Source:http://en.wikipedia.org/wiki/Star_network

[5] OPNET Technologies, Inc. OPNET Modeler Product Documentation, release 118.

[6] OPNET Technologies, (2008) Basics of OPNET IT Guru Academic Edition,

[7] Guo, J., Xiang ,W., and Wang, S., (2007).Reinforce Networking Theory with OPNET Simulation, Journal of Information Technology Education, Volume 6.

[8] OPNET Technologies, (2004).The World's Leading Network Modeling and Simulation Environment.

[9] Dolejš, O., OPNET modeler - networks simulation Department of Control Engineering ,Czech Technical college.

[10] Kaur, B.,( May, 2012)OPNET based Analysis of Star Topology in Small Internetworks" International Journal of Electronics \& Data Communication www.cirworld.com Volume1 No. 1. 